\title{
Design of State Rail and Bus Transportation Scheme with Bi-Level Optimization Model
}

\author{
K. Pavlova, T. Stoilov
}

Key Words: Optimization; networkdesign; bi-level optimization; rail public transportations.

Abstract. The increase of the rail public transportations is searched in directions for redistribution of the passenger travels between rail and bus transportation. The rail transport benefits by increasing it schedule for places where the transportation capacities on appropriate directions is not achieved. A mathematical model has been derived to assess the potential of the rail passenger transport to increase his capacity and efficiency. This potential has been evaluate in comparison with the competition of the bus transportation. A specific transportation route has been chosen from Sofia to Varna and the potential for increase of the rail transport has been evaluated. The mathematical model uses bi-level optimization problem, related to the evaluation of a maximal flow in a transportation network.

\section{Introduction}

On National level bus and rail transports are main means of usage for travelers. But the European practice gives preferences for travailing by rail. Thus providing state national transportation scheme, supporting the long journeys by rails is an efficient and advance policy for transport. Thus the rail passenger transportation can be an important player for the development of Intelligent Transportation Systems. The rail transportation is giving advantages due to it benefits, concerning efficiency in exploitation, improving logistics services, reducing the pollution. This paper tries to give priority to rail transport among the Bulgarian National Transport Scheme. This scheme is maintained by both bus and rails means of transportation. On National level the Ministry of transport is entitled to provide resources to rail and bus operators to exploit the different components of the National Transport Scheme. Together with the task for implementation of this scheme, the ministry of Transport has to provide National policy for the development of the rail transportation as important and priority transport system. Thus on National Level a problem arises for priority support and implementation of the rail transportation and simultaneously to insure operability of the National Transportation Scheme. The trivial approach is to provide bus transportations only on place where rail transportation is missing. But capacity restrictions on rail system cross connections between bus and rail transportation routes, common stops in towns for bus and rail provide competitions between the rail and bus transport services. In such competition environment the National rail policy insists to give preferences and increase the exploitation of the rail transport. This paper tries to formalize the problem for increase the priority of rail transport. The formal description of this problem is to provide an optimal allocation of the rail transportation services over the National Transportation Scheme, taking advantage from the bus transport. An optimization problem is defined, which generate a solution for increase of the rail transport. The optimization problem is defined in bi-level form. The upper optimization problem evaluates the maximal flow, which can be passed between two predefined points in the National Transportation Scheme. The maximal flow is implemented both by bus and rail transport according to the predefined capacities for transportation on different transport links. On the lower level the optimization problem evaluates the low cost flow distribution by minimization of the transportation costs $[4,6]$. The rail transport has advantages for less cost in comparison with the bus transport. The flows per different link are used as capacities for the upper level problem. As a result the bi-level problem gives solution both by maximization of the transport flow between two predefined important towns in Bulgaria and optimizing the parts of the maximal flow, performed by rail.

The paper makes an illustration in definition and solution of a transportation problem by bi-level optimization. The hierarchical order is applied for two optimization problems. The paper doesn't contain developments of new algorithms for solution of bi-level problem, but it contributes for the increase of the application area of this more complex optimization formalism $[3,5]$.

\section{Objectives and Tasks of the Article}

The aim of the paper is to develop a formal model and algorithms for its application in solving a research task for intensification the railway passenger transport, using a section of the Bulgarian National Transport Scheme.

The subject of the survey is an integrated transport system including passenger transport by rail and bus transport.

A two-hierarchy model for the optimization of rail passenger transport is used. A management policy is created that uses prediction coordination, the task from the top level is to find a maximum flow, and the task at the bottom level is to find the lowest cost flow distribution. To develop a bi-level model, to define a two-hierarchical optimization problem, to formalize the solution of the problem as management policy for railway passenger transport 
concerning intensification of rail transport, the paper solves the following tasks:

- Development of hierarchical model for managing an integrated transport system. The model should give priority to rail passenger transport compared to bus transport.

- The hierarchical model should take advantage by decomposition of the hierarchical model through interrelated optimization subproblems.

- Design of algorithm for quantification the parameters for the hierarchical problem concerning the estimation of the traffic capacities of the transportation links.

- Analytical definition and solution of the hierarchical optimization problem and assessment of the solutions.

- Application and interpretation of the hierarchical model for increasing the rail public transportation.

\section{Case Study}

In 1955 the US Air Force developed the project RAND addressing the evaluation of the rail net capacities of the West part of Soviet Union and the satellite countries in Europe. This project, developed by T. Haris and F. Ross motivated Ford and Fulkerson to provide researches and to develop formal descriptions and algorithms for evaluation of the maximal flow in a network. The problem, which was raised by [2] was defined as: given the capacities of individual links in a rail network how can be evaluated the over-all capability of the entire rail network. The researches in [2] targeted a way to define the minimum cut of the Soviet rail system, which is needed by the US air forces. In [4,9] the minimal cut problem was defined in current form as maximal flow in a network. It is well known currently that the maximal flow is equal to the minimal cut of the network. An extended history for the origins of this theoretical result can be found in [2].

The "maximal flow" problem can be assumed as problem for analysis of a network system. The optimization problem can be defined in the form: given a topology of a network with capacities values on the links of the network. How much is the maximal amount of flow between two nodes, which can be passed through the network.

The analytical definition of the maximal flow problem is presented in the form

$$
\begin{aligned}
& \max _{f_{i j}}\left[f_{s t}\right] \\
& \sum_{j \in A(j)} f_{i j}-\sum_{j \in B(j)} f_{j i}=\left\{\begin{array}{l}
0, i \neq s, t \\
f_{s t}^{*}, i=s \\
-f_{s t}^{*}, i=t
\end{array}\right. \\
& f_{i j} \leq v_{i j},(\forall i, j) \in N
\end{aligned}
$$

where $i, j$ denotes number of nodes in a network with $\mathrm{N}$ nodes.

Relation (1b) describes the continuity nature of the flows which enter and outgo to/from a node. Relation (1c) insists the flows on a link to respect the link capacity, which is predefined and constrained. The goal function (1a) de- scribes the requirement for maximization of the flow $f_{s t}$ which starts from the initial node $\mathrm{s}$ and enter to the final one $\mathrm{t}$.

The solution of problem (1) gives the value $f_{s t}$ of the maximal flow, which can be transmitted between the network from $\mathrm{s}$ to t. Additionally the values of $f_{i j}$ define the paths which the maximal flow uses through the network.

Hence for given capacity on the network links, the maximal flow problem (1) gives the amount of flow, which can pass through the predefined network topology.

Another corresponding optimization problem, related to the network design is defined as "minimization cost network problem". This problem considers the flow distribution on network. But it defines the value of the link capacities $v_{i j}$, which correspond to the values of the flow components , $v_{i j} \geq f_{i j}$, and minimizing a cost function. The analytical form of the "minimum cost problem" on linear form can be written as:

(2a)

$$
\min _{x_{i j}} \sum_{i j \in A} c_{i j} f_{i j}
$$

$$
\sum_{j \in A(j)} f_{i j}-\sum_{j \in B(j)} f_{j i}=\left\{\begin{array}{l}
0, i \neq s, t \\
f_{s t}{ }^{*}, i=s \\
-f_{s t}{ }^{*}, i=t
\end{array}\right.
$$$$
\text { (2c) } \quad a_{i j} \leq f_{i j} \leq b_{i j},(\forall i, j) \in N
$$

where

$f_{i j}$ are the values of the flows on arks between nodes $\mathrm{i}$ and $\mathrm{j}$;

$c_{i j}$ are the cost for transmitting a unit flow between nodes $\mathrm{I}$ and $\mathrm{j}$;

$a_{i j}$ and $b_{i j}$ are the lower and upper boundaries of the flow between nodes $\mathrm{I}$ and $\mathrm{j}$;

$f_{s t}$ is the flow, which has to be transmitted from node $s$ to $t$ by minimizing the transportation costs (2a).

Relations (2b) correspond to the equations for the conservation the flow. The solution of (2) gives optimal flow distribution $f_{i j}$. The values of $f_{i j}$ define the minimal capacities of the links by means to provide the cheapest flow distribution. A verification of problem (2) related to the design of network topology is introduced by [7].

This paper started with evaluation of the components of the maximal flow between two towns: Sofia and Varna. The maximal flow is evaluated in a network, representing the integral transportation links of the National Network, supported both by bus and rail transports. This evaluation of maximal flow and it components allows to identify solutions for intensification of passenger rail transport; to evaluate opportunities to improve the economic performance of the system of passenger rail transport; to make quantitative assessments of operation of the rail passenger to identify measures to intensify the rail transport and compliance with European directives (Directive 2010/40/EU of European Parliament and of the Council of 7 July 2010 on the framework for the deployment of Intelligent transport Systems in the field of road transport and for interfaces with other models of transport), to reduce pollution from emissions and improving logistics services through intensification of 
rail transport. For the development of such mathematical model for improvement and intensification of the rail transport hierarchical approach is used by means to interconnect and solved multilevel optimization problems. For the particular case the paper applies definition and solution of bilevel optimization $[1,3,4]$.

The idea of the multilevel optimization approach assumes that on each hierarchical level the corresponding subsystem solves appropriate optimization subproblem. The solutions of this subproblem influence the upper and the lower levels optimization subproblems. Thus an interconnected set of optimization subproblems have to be defined and solved. The multilevel technology of optimization allows to be hierarchically order set of goal function, to be considered wide set of constraints. The overall optimal solutions contain wider set of solutions. The drawback of the multilevel optimization origins from the complexity of such problems. The complexity contains necessity to find global optimum, to deal with nonconvex and not differentiated functions.

This research applies bi-level formalism for the optimization of the rail transportation. On the lower level the maximal flow in network is defined, where the capacities of the links are predefined. The upper level evaluates minimal cost flow finding optimal capacities on the links. By the interconnection in bi-level definition these two problems give optimal flow distribution in the network, giving preference for the flows to the cheapest transportation.

\section{Formal Model of Optimal Traffic Distribution in Network by Bi-Level Optimization}

Important parameter for the optimization problem is the value of traffic, which has to be transported between two nodes of the network. For the case of multiple pairs of Origin-Destination points an OD matrix has to be estimated. The practical considerations insist that the network has to be designed in a way that between each OD pair the transportation flow has to be maximal. This problem is titled as Demand Adjustment Problem (DAP) [6,10]. The formal description of DAP problem is the following. For a given topo$\operatorname{logy}$ of the network $\mathrm{R}=(\mathrm{N}, \mathrm{A})$, where $\mathrm{N}$ is the set of nodes, $\mathrm{A}$ is the set of links the following notations are used:

I is a set with the pairs of nodes for Origin-Destination transportation services;

$v=\left(v_{a} \mid \alpha \varepsilon \mathrm{A}\right)$ vector where each component $v_{a}$ is the flow volume which pass trough link $\alpha$;

$h=\left(h_{k} \mid k \varepsilon K . i \varepsilon I\right)$, defines a set of components $h_{k}$ which is the flow between k-th pair of nodes in the network;

$K_{i}$ is the set of all pair nodes in I;

$h$ is the total volume of flow q passing in the network; $g=\left(g_{i} \mid i \varepsilon \mathrm{I}\right) \varepsilon G$ is a matrix, defining the corresponding flow volumes, which have to be exchanged between each pair of $\mathrm{O}-\mathrm{D}$ nodes; $\mathrm{G}$ is finite set of values. The flow distribution h, which satisfies the required flow matrix $g$, corresponding to the $\mathrm{O}-\mathrm{D}$ matrix, defines the values $\mathrm{v}$ of the flows, which pass to each links of the network. $[4,8]$

For the DAP problem, the solution is the volume $g$ of the flow, which is distributed on each link of the network. The problem DAP can include additional constraints for example to consider the costs for transportation of flows per different links. The cost value can be a function of the flow $\mathrm{v}$, which pass per the corresponding link, $s_{a}(\mathrm{v})$. The total cost for transportation of traffic volume $h$ in the network is noted as $s_{k}(\mathrm{~h})$ [8].

The simplest method for solving DAP assumes that the volume of traffic, which passes through a link doesn't change it capacity. For that case the DAP problem is formalized with the linear relations

$$
\sum_{i \in I} p_{i a}(g) g_{i}=v_{a}^{\prime}
$$

where $p_{i a}$ is the set links, which are used by the flow $g_{i}$ in the network and respecting the constraint for the link capacity $v_{a}^{\prime}$.

The optimization form of DAP problem defines a goal function for maximization of the total amount of flows $g$ $\max F\left(g, g^{\prime}\right)$

(3) $\sum_{i \in I} p_{i a} g_{i}=v_{a}^{\prime}, a \in A$ $g_{i} \geq 0, \mathrm{i} \in \mathrm{I}$

The problem (3) evaluates the maximal flow volume $g$, which can be transported through the network, satisfying the capacity constraints $v_{a}^{\prime}$ for the links in the network, $a \in A$.

It is possible problem (3) to lack of solutions due to the empty feasible set of constraints. For practical applications, facing such situation needs to change the problem by using another set of capacity volumes $\mathrm{v}$.

In a common case, problem (3) can have goal function both for the flows $g$ as for the capacity volumes $v$ :

$$
\begin{aligned}
& \min \gamma_{1} F_{l}\left(g, g^{\prime}\right)+\gamma_{2} F_{2}\left(v, v^{\prime}\right) \\
& \text { (4) } \sum_{i \in I} p_{i a} g_{i}=v_{a}, a \in A \\
& g_{i} \geq 0, \mathrm{i} \in \mathrm{I}
\end{aligned}
$$

where the notation $F_{2}\left(v, v^{\prime}\right)$ can formalizes requirements for the capacity values between reference values and the problem solutions. The same meaning of the function $F_{l}\left(g, g^{\prime}\right)$ can be requested to keep in correspondence the referee values of the flows $g$ and the problem solutions.

This research introduces a specific form of problem (4). The application area of the problem is to redistribute the transportation flows in a network, by means to redirect the optimal flows to predefined links in the network. The network is constituted by the passenger transportation services, undertaken both by rail and bus transports. The predefined links, which have to be undertaken the main traffic are these ones, belonging to the rail transport.

\section{Estimation of the Capacities of the Links}

Due to the lack of data about the passenger flows in the 
transportation network, this research develops an approach for using the time travel on each link as a value for assessment the capacities of them. At first it has been identified each transportation bus line, which crosses the rail path through Sofia to Varna. Such identification resulted in definition of a graph, given in figure 1 .

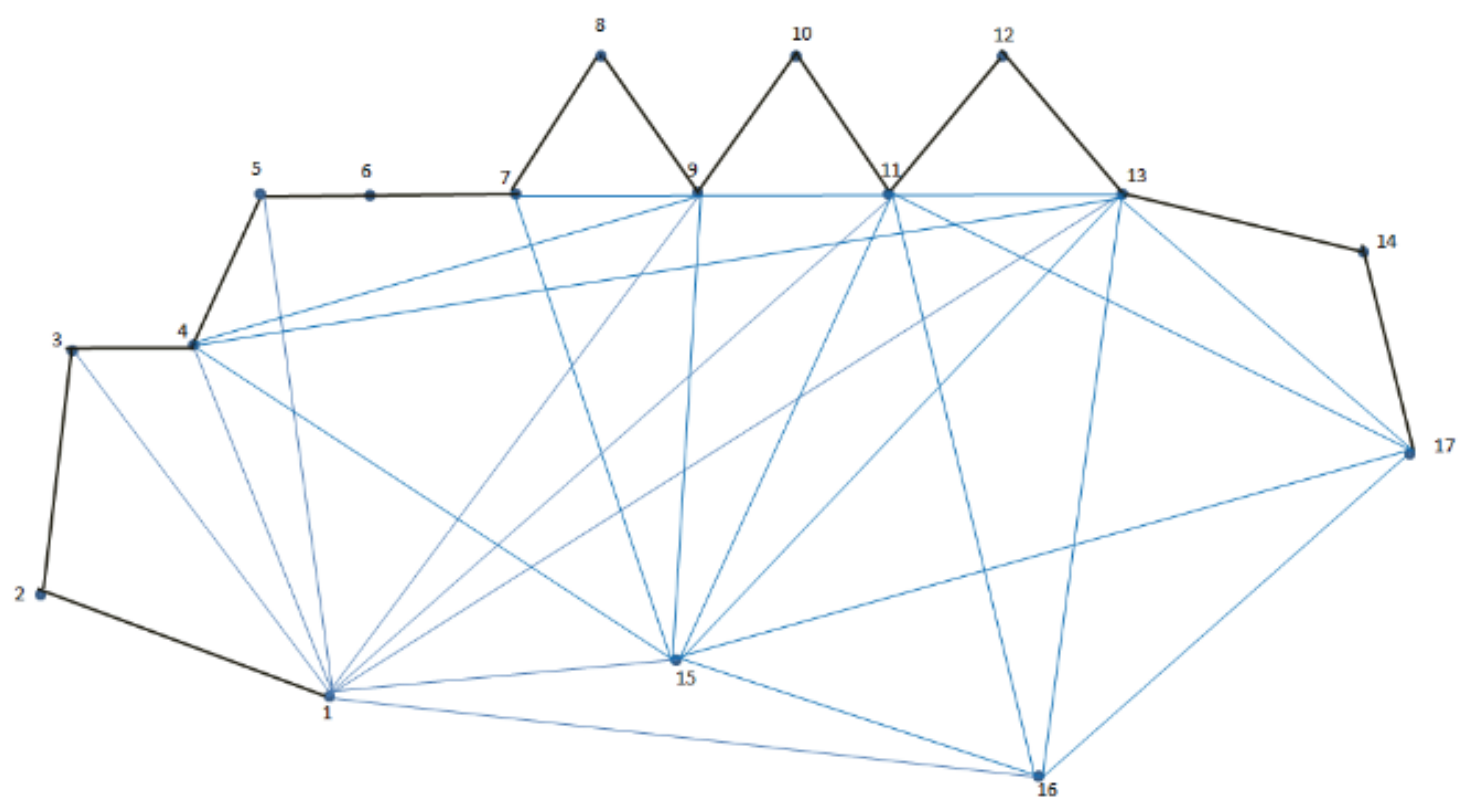

Figure 1. Topology of the network

For each links of this graph a capacity value was allocated. The considerations which have been applied address that the passengers preferences for fast traveling.

Hence if the time for travelling per link $i$ takes time $t$, this paper assumes that the capacity for flow transportation per link $i$ is equal to
(5) $v_{i}=1 / t$

The transport network was estimated for the real case between two important towns in Bulgaria: Sofia and Varna. The input data for the rail transportation from Sofia to Varna was taken from the real operating schedule, presented online in www.bdz.bg, figure 2.

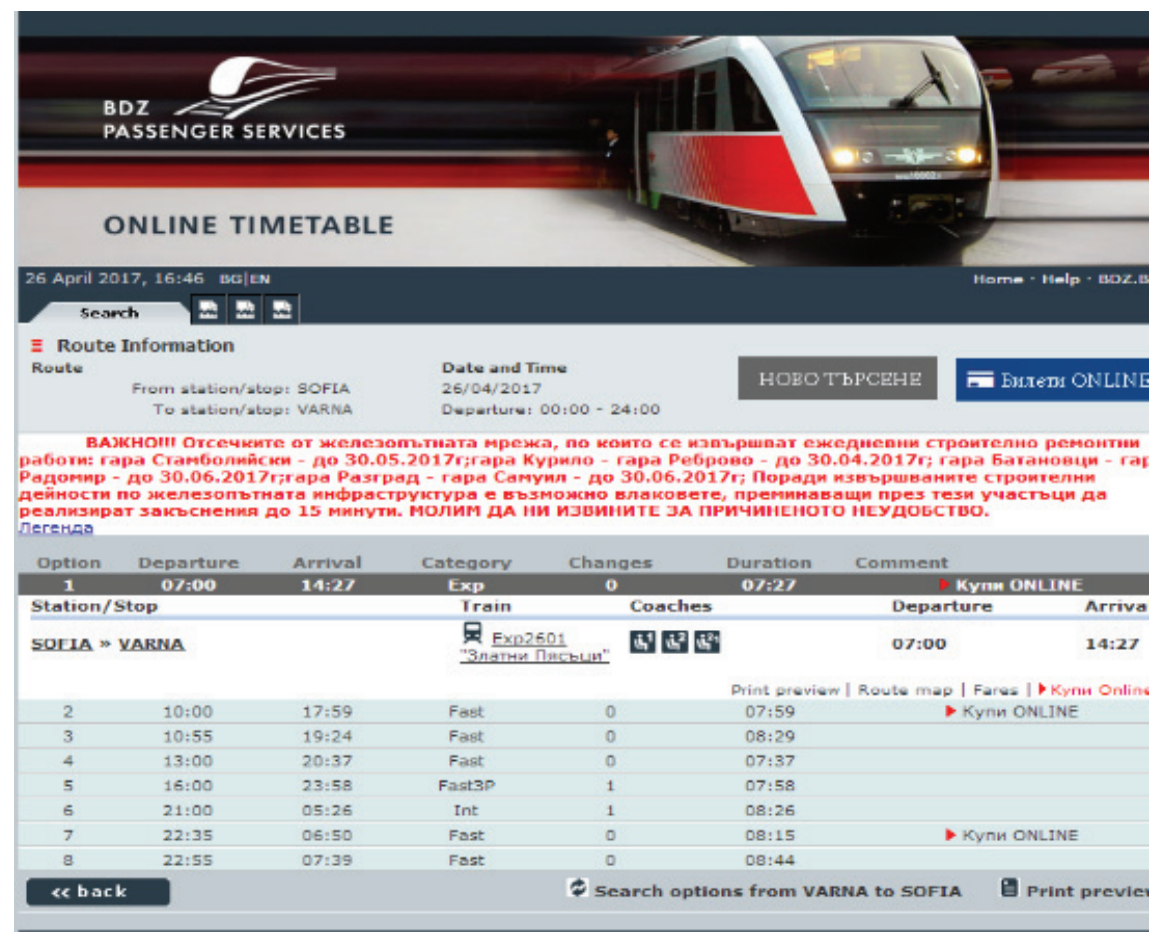

Figure 2. The current schedule of trains between Sofia and Varna 
Following the rail schedule it has been identified 17 stops which define the network structure with 17 nodes. The actual lines for bus transportation were taken from the Ministry of Transport, Information Technology and Communications, which issues the licensees for bus transportation, publically available in http://rta.government.bg/ index.php?page $=$ scategories \&scategory $=$ otrts.

Applying relation (5), the travel time by rail from Sofia to Levski is 3 hours and 23 minutes or 203 minutes. Respectively the flow capacity on this link is $1 / 203=0.0049$ relative capacity units. On daily basis on this link three times rail is passing, which means that the total capacity of this link for flow transportation will be: 3 times x 0.0049 capacity units $=0.014$ capacity units for this link.

By the same way, using the schedules on each bus and rail link it has been estimated the links capacities for the all network of figure 1. Having these capacities values, the DAP problem has been defined analytically and solved.

\section{Definition of the First Optimization Problem}

$$
\begin{aligned}
& \max X\left(f_{i}^{k}\right) \\
& \sum_{i} f_{i}^{k}-\sum_{j} f_{j}^{k}=0 \\
& \sum_{i} f_{i}^{s}=-X \\
& \sum_{j} f_{j}^{d}=X \\
& \sum_{i} f_{i}^{k} \leq V_{i, k}
\end{aligned}
$$

The task determines the values of flows $f_{i j}$ and the corresponding arcs on which the maximal flow is generated.

The optimization problem contains 44 variables, 17 constraints for flow equilibrium for each of 17 nodes in the network and 44 upper bounds constraints. The problem has been solved, applying a MATLAB function graphmaxflow(). The solution of the optimization problem is graphically presented on figure 3.

The town notations correspond as follows:
1. Sofia
2. $\mathrm{x}$
3. Mezdra
4. Pleven
5. Levski
6. Gorna Orahovica
7. Strajica
8. $\mathrm{y}$

9. Popovo

10. z

11. Targovishte

12. $\mathrm{W}$

13. Shumen

14. $\mathrm{t}$

15. V. Tarnovo

16. Antonovo

17. Varna

$\mathrm{x}, \mathrm{y}, \mathrm{z}, \mathrm{t}$ are mock points by means to lack the overlapping of the rail and bus transport on a same link.

The graphical representation of DAP problem solution, figure 3, illustrates the maximal flow, which can be transmitted between Sofia and Varna. The maximal flow has subflows, which pass through different routes. Thus the network links has different utilization of their capacities.

To each link in the transport network a fraction numbers is given. The numerator presents the value of flow, which is a part of the max-flow, which passed through this link. The denominator is the capacity of the link:

$$
\frac{\text { numerator }}{\text { denominator }}=\frac{\text { component of } \max \text { flow }}{\text { capacity of link }}
$$

The solution of problem (6) gives value of the maximal flow, which can be transmitted from node 1 to node 17 (from Sofia to Varna). The relative value of this flow is 0.2485 relative flow units. Making comparisons for each link it can be identified, that the capacities of links between nodes $1-2,2-3$, are totally used by the components of the max flow. Hence additional traffic between nodes 1 and 17 is not possible to be allocated. Respectively this result proves decisions for the rail authorities to increase the capacities of rail transportations for these directions. This can be done by increase of the frequency of schedules for trains to these directions.

From figure 3 it is evident that a set of links, supported by rail transportation have capacities, which currently are not used. This give potential to redistribute the traffic flows by means to increase the rail utilization and to decrease the bus transport. Such policy can be implemented by definition and solution of additional optimization problem. The last will evaluate the cheap flow distribution, applying lower costs for rail transport according to the bus transportation.

This additional optimization problem has the form (2). The particular case is that this problem has to respect the value of the maximal flow, which was estimated by the solution of problem (6). The requirement for low cost distribution on rail links and transmitting max-flow through the network leads to analytical definition of the bi-level optimization problem. 


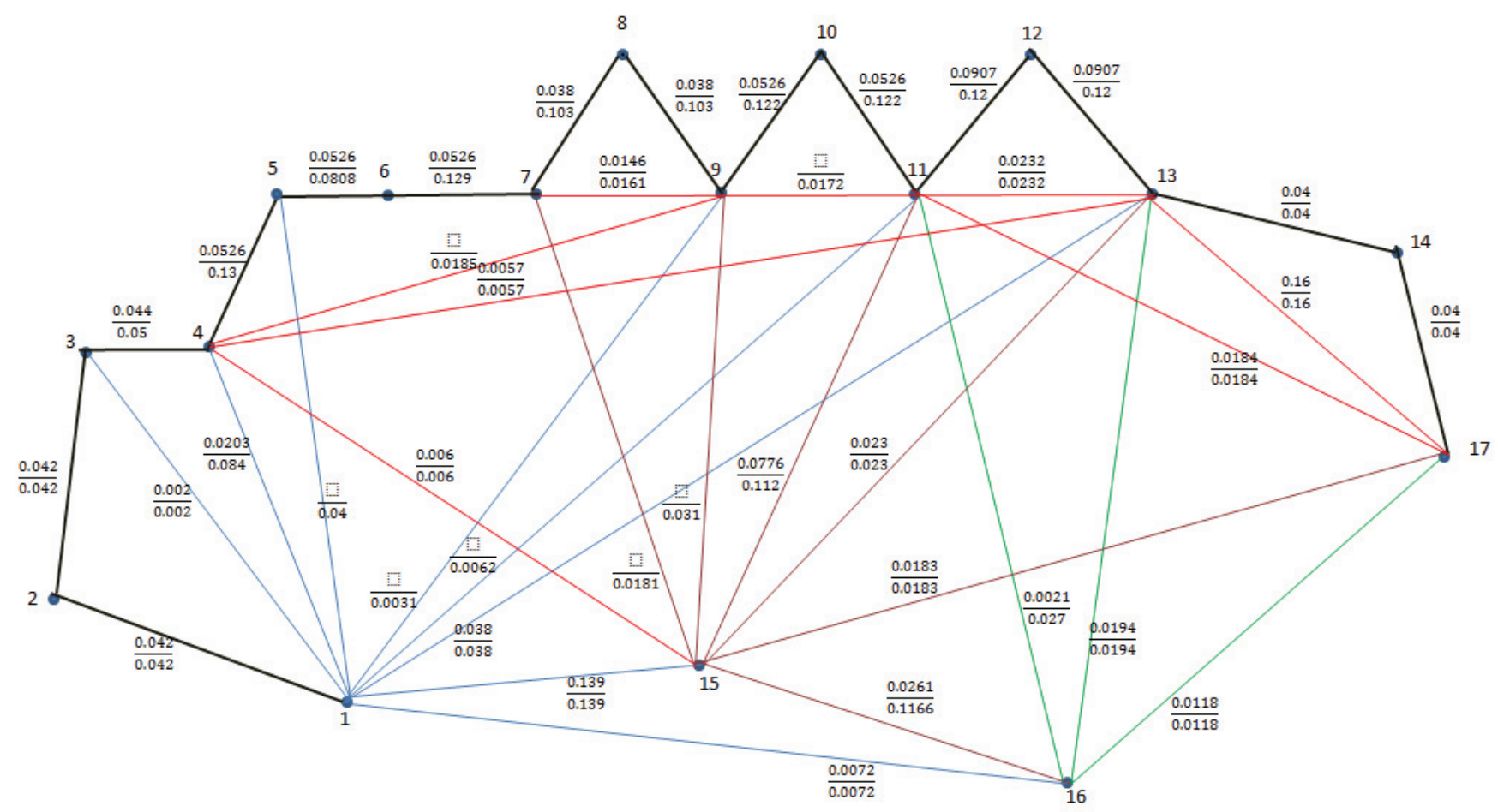

Figure 3. Graphical solution of the max flow problem

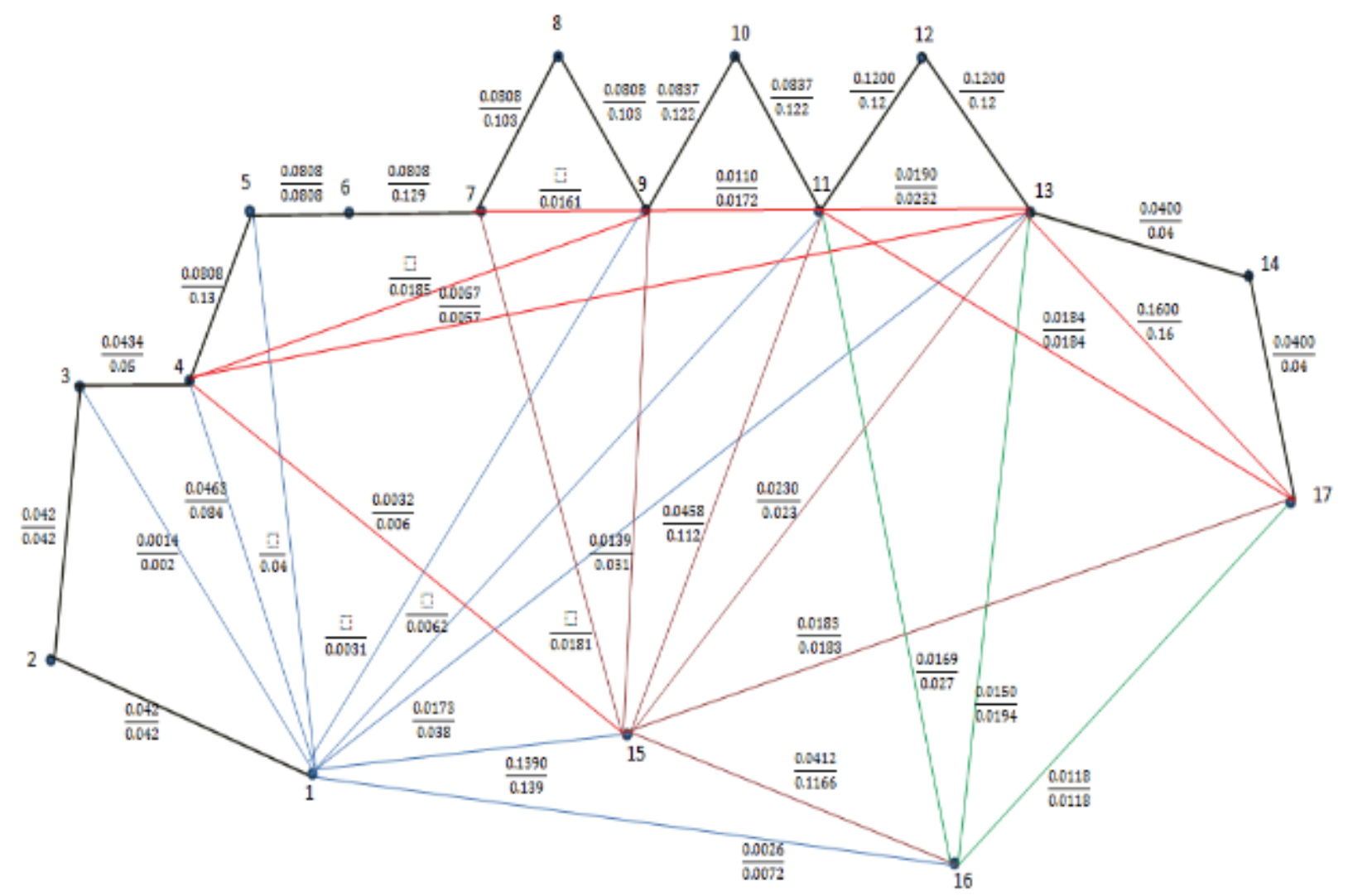

Figure 4. Graphical solution of the bi-level problem 


\section{Traffic Flow Distribution According to Bi-Level Optimization Problem}

The upper level problem evaluates the maximal flow, which can be transported between nodes 1 and 17 . The components of max-flow have to respect the initial capacity restrictions of the links. As a result the value of the max flow is given as a parameter to the lower optimization problem, defined as minimal cost flow distribution (2) $[4,6]$.

The minimal cost distribution problem evaluates the flows, which give preferences to the rail transport. These new values of flow distribution are passed to the upper optimization problem as new capacity restrictions for the max flow problem. The solution of the bi-level problem formally is presented on figure 4.

The numerical solution of the bi-level optimization problem has been done by MATLAB suit. The evaluations have been performed with an additional function for solving bi-level hierarchical tasks, solvebi-level (). This function does not enter into the MATLAB program configuration. It has been downloaded by the YALMIP project [10].

The comparisons between the results of max flow and the bi-level problem figures 3 and 4, demonstrates that the bi-level problem increases the usage of the rail transportation. For example the flow on link between nodes 5-6 increases from 0.0526 to 0.0808 , which is the value of the available capacity of this link. Such increases are met additionally for a set of rail supported links:

Link (5-6): increase from 0.0526 to 0.0808

Link (6-7): increase from 0.0526 to 0.0808

Link (7-8): increase from 0.038 to 0.0808

Link (8-9): increase from 0.038 to 0.0808

Link (9-10): increase from 0.0526 to 0.0887

Link (10-11): increase from 0.0526 to 0.0887

Link (11-12): increase from 0.0907 to 0.12

Link (12-13): increase from 0.0907 to 0.12

The increase of the rail network links is accompanied by decrease of transportation on bus links:

Link (1-13): decrease from 0.038 to 0.0173

Link (5-6): decrease from 0.0146 to 0

Link (5-6): decrease from 0.0232 to 0.0190

These results prove that the bi-level optimization problem can additionally take into consideration not only the maximization of the flow between two points into the transportation network, but to provide additional minimization of the bus transportation. Particularly this research gives benefits to the rail transport, which is a formal and optimal way to prove policies for intensification of the rail public transportation.

\section{Conclusions}

This research extends the bi-level optimization to a new domain of applications. It has been defined a real optimization problem, which increases the exploitation and transportation of rail transport. It gives formal basis for assessment of the policy for providing licensees for bus transportation. The formal model for increase of rail transport founds on the integration of problems for maximization of the transport flow between two points into transport network and minimization of the cost of traffic flows. Thus the bi-level problem gives solution both for rail and bus transportation and takes into considerations requirements for these general types of transportation in a National Transport Scheme. The solutions present the weak points, where the rail infrastructure has to be improved, suggest where to intensify more frequently the rail schedules, how to provide national policy for giving licensees for bus transportation.

The parameters of the bi-level problems have been estimated according to real transportation schedules in Bulgaria. The results, presented can be used for practical implementation of the bi-level transportation problem for other related cases for providing priorities on different types of services.

\section{References}

1. Brotcorne, L., M. Labbe, P. Marcotte and G. Savard. A Bilevel Model and Solution Algorithm for a Freight Tariff Setting Problem. - Transportation Science, 34, 2000, 289-302.

2. Cormen, T., Ch. Leiserson, R. Rivest, S. Clifford. Section 26.2: The Ford-Fulkerson Method. Introduction to Algorithms (Second Ed.). MIT Press and McGraw-Hill. 2001, 651-664, ISBN 0-262-03293-7.

3. Dempe, S. Foundations of Bilevel Programming. Kluwer Academic Publishers, Dordrecht, 2002.

4. Kalashnikov V., S. Dempe, G. Alfredo Perez-Valdes, José-Fernando Camacho-Vallejo. Bilevel Programming and Applications, March 2015, Mathematical Problems in Engineering 2015(2): 1-16, DOI:10.1155/2015/310301.

5. Heineman G., G. Pollice, St. Selkow. Chapter 8: Network Flow Algorithms. Oreilly Media. 2008, 226-250, ISBN 978-0-596-51624.

6. Kleinberg J., É. Tardos. (2006). Chapter 7: Extensions to the Maximum-Flow Problem. Algorithm Design. Pearson Educ. 378-384. ISBN 0-321-29535-8.

7. Lasdon, L. Optimization Theory For Large Scale System. Mcc. Milan, N.Y.., 1970

8. Sharminda, B., K. Rao. Estimation of Origin-destination Matrix from Traffic Counts: the State of the Art. European Transport, n. $49,2011,3-23$.

9. Shigeno, M. A Survey of Combinatorial Maximum Flow Algorithm on a Network with Gains. - Journal of Operation Research, 47, 2004, 244-264.

10. https://yalmip.github.io/command 
Manuscript received on 08.12.2017

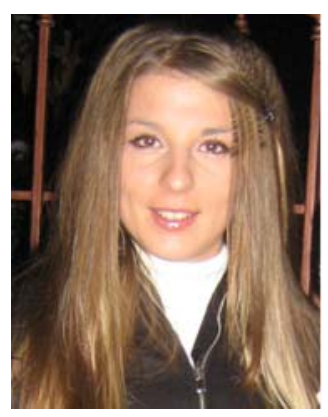

Kristina Pavlova graduated from University of Chemical Technology and Metallurgy - Sofia, Bulgaria, Specialty Automation and Information Technologies in 2013. She received a PhD in 2017. Her research interests are in area of application of optimization for system control and design. Particular developments address bi-level optimization in hierarchical and complex systems as distributed systems, transportation control systems, real time software design. She has research achievement in projects, funded by the National scientific fund, Bulgarian Academy of Sciences, Bulgarian small and medium enterprises promotion agency.

Contacts:

Institute of Information and Communication Technologies Bulgarian Academy of Sciences, 1113 Sofia, Bulgaria Acad. G. Bonchev St., bl. 2, tel. +35929792774 e-mail: kristina@hsi.iccs.bas.bg

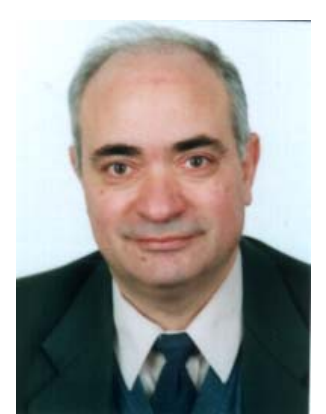

Todor Stoilov received a Ph.D. in 1979 and Doctor of technical sciences on 1999. Since 2000 he is a full professor in Bulgarian Academy of Science. His researches address complex control and hierarchical systems as transportation systems, communication networks, economic processes, software design of real time information services. His formal domain is the definition, solution and application of optimization problems, particularly for multilevel optimization. He has published about 350 papers, includes four monographs in Bulgaria and one abroad. For two IFAC terms he has been elected and has chaired IFAC Technical Committer 7.4 "Control of transportation systems". His activities are well known by the academic audience in Europe, according his management in EU projects since FP 4, 5, 6, 7, and Cost programs.

Contacts:

Institute of Information and Communication Technologies Bulgarian Academy of Sciences, 1113 Sofia, Bulgaria Acad. G. Bonchev St., bl. 2 e-mail: Todor@hsi.iccs.bas.bg 